\title{
Corrigendum: Book Review: The Big Five in SLA
}

\author{
Yuanyuan Miao* \\ School of Foreign Studies, Guangzhou University of Chinese Medicine, Guangzhou, China
}

Keywords: second language acquisition, neuroticism extraversion, openness to experience, agreeableness, conscientiousness

\section{A Corrigendum on}

OPEN ACCESS

Approved by:

Frontiers Editorial Office,

Frontiers Media SA, Switzerland

${ }^{*}$ Correspondence:

Yuanyuan Miao

miaoyuan@gzucm.edu.cn

Specialty section:

This article was submitted to

Educational Psychology,

a section of the journal

Frontiers in Psychology

Received: 21 July 2021

Accepted: 26 July 2021

Published: 16 August 2021

Citation:

Miao Y (2021) Corrigendum: Book

Review: The Big Five in SLA.

Front. Psychol. 12:745119.

doi: 10.3389/fpsyg.2021.745119
Book Review: The Big Five in SLA

by Miao, Y. (2021). Front. Psychol. 12:710042. doi: 10.3389/fpsyg.2021.710042

In the original article, a funder was neglected, Guangzhou Social Science Planning Co Construction Project in 2021-Study on the Multi-Humanistic Path of the Overseas Spread of Traditional Chinese Medicine from the Perspective of Systematic Philosophy (No: 2021GZGJ97).

In the published article, there was an error in the affiliation. Instead of "School of Foreign Studies, Guangzhou Medical University,Guangzhou, China," it should be "School of Foreign Studies, Guangzhou University of Chinese Medicine, Guangzhou, China."

The author apologizes for this error and states that this does not change the scientific conclusions of the article in any way. The original article has been updated.

Publisher's Note: All claims expressed in this article are solely those of the authors and do not necessarily represent those of their affiliated organizations, or those of the publisher, the editors and the reviewers. Any product that may be evaluated in this article, or claim that may be made by its manufacturer, is not guaranteed or endorsed by the publisher.

Copyright (c) 2021 Miao. This is an open-access article distributed under the terms of the Creative Commons Attribution License (CC BY). The use, distribution or reproduction in other forums is permitted, provided the original author(s) and the copyright owner(s) are credited and that the original publication in this journal is cited, in accordance with accepted academic practice. No use, distribution or reproduction is permitted which does not comply with these terms. 